\title{
INTERFACIAL INSTABILITY OF DENSITY-STRATIFIED TWO-LAYER SYSTEMS UNDER INITIAL STRESS
}

\author{
N. TRIANTAFYllidis \\ Department of Aerospace Engineering, University of Michigan Ann Arbor, MI 48109-2140, U.S.A. \\ and \\ F. K. LEHNER \\ Koninklijke/Shell Exploratie en Produktie Laboratorium, 2288 GD Rijswijk Z.H., The Netherlands
}

(Received 8 May 1991 ; in revised form 13 February 1992)

\begin{abstract}
THE ONSET of interfacial instability in two coherent semi-infinite layers of different properties and the corresponding critical wavelength are found by solving a static bifurcation problem in finite plane strain. Subsequently, the stability of perturbations of any wavelength is determined from the appropriate linearized equations of motion. For gravitationally stable or unstable density stratifications, the critical stress at which the interface is destabilized is shown to depend on the wavelength of a perturbation; it is also determined in a complex manner by initial stress gradients perpendicular to the layer interface and by layer stiffness, as is illustrated here in detail for the examples of a hyperelastic solid and an elastoplastic solid, both resting on an inviscid fluid of different density. The very large wavelength that is predicted for the gravitational instability of a semi-infinite elastic solid on a buoyant fluid substratum brings forward the essential role of pre-stress and associated stiffness reduction as well as that of a finite layer thickness in destabilizing geological and geophysical two-layer systems.
\end{abstract}

\section{INTRODUCTION}

A VARIETY of problems lead to the question of the precise conditions under which an initially plane interface between two layers of different properties may be destabilized by layer-parallel stresses, counteracted or aided in their effect by body forces. An example that has been of particular interest to geologists and geophysicists is the instability of density-stratified two-layer systems in a gravitational field [see e.g. RAMBERG (1981)] and the same question has motivated the present investigation. Earlier theoretical studies of this problem have mostly been concerned with the Rayleigh Taylor model of two supcrimposed viscous fluids, in which gravity provides the only destabilizing force. In the classical stability analysis of this problem (TAYLOR, 1950; Chandrasekhar, 1955, 1961 ; Daneš, 1964 ; Selig, 1965) the initial growth of a small interfacial perturbation is studied. Although all wavelengths are found to satisfy the linearized perturbation equations, the presence of viscosity implies the existence of a dominant wavelength that possesses the fastest growing amplitude and is therefore expected to characterize the evolution of the system. However, an essential limitation of fluid dynamical models of interfacial stability has been their inability to

(C) 1992 Shell Research B.V. 
account for the effects of layer stiffness and the stabilizing or destabilizing role of anisotropic and nonhydrostatic states of stress. An entirely different approach to the problem of surface as well as interfacial instability in laterally compressed elastic and viscoelastic materials was pioneered by BIOT (1963a, b, c ; 1965), who treated the onset of interfacial instability as a bifurcation problem.

This paper seeks to extend Biot's analysis by considering the combined effects of gravity and nonhomogeneous states of initial stress on interfacial instability; its aim is to establish conditions for the existence of a nontrivial solution to the incremental equilibrium equations of two solid material half-spaces of differing densities that are separated by an initially planar interface normal to the direction of gravity and are allowed to undergo finite deformation in plane strain. An analytical condition will be given that relates the critical wavelength for a bifurcation to the material properties and stress states in each half-space. The modes of interfacial instability that are obtained from the following analysis exclude an entire range of wavelengths and-for a fixed load--imply a single admissible critical wavelength.

A second aim of this paper is to study the stability of the layer interface under the assumed general conditions. The stability of interfacial disturbances of arbitrary wavelength will be characterized by means of a perturbation analysis of the system's linearized equations of motion. A perturbation is characterized as unstable if its amplitude increases with time; periodic perturbations of constant amplitude are to be considered stable for reasons explained in Section 2. Finally, perturbations which, according to a linear analysis, exhibit neutral stability with time-independent perturbation modes are shown to correspond to the critical wavelength. The connection between bifurcation and stability is thereby established in a quantitative way.

For the case of a gravitationally unstable density stratification and in the absence of a gradient in prestress, perturbation modes below the critical wavelength are found to be stable while those above the critical wavelength are found to be unstable as expected. Indeed, perturbation modes below a certain wavelength require more work than can be extracted from the system by lowering its potential energy; these modes are therefore inhibited. The critical wavelength is that which balances the two energies and allows the incremental equilibrium equations for the corresponding perturbation mode to be satisfied. The situation is considerably complicated by a gradient in prestress, as a consequence of which the results become more sensitive to the nonlinearity of the constitutive relation.

Following the development of the general theory, an application will be made to the case where the lower half-space is occupied by an inviscid fluid and the upper by a solid of different density, both materials being taken as incompressible. The effects of non-linear constitutive behaviour will be explored by contrasting the stability behaviour of a rubber-like solid with that of a rate-independent elastoplastic material whose stiffness decreases with deformation.

\section{Governing EQuations}

The instability of a pre-stressed heavy layer resting on a substratum of different density will be analysed in the following as a static bifurcation phenomenon. The 
onset of the bifurcation, i.e. the instance of loss of uniqueness of the trivial principal solution for a flat interface, will be studied first. This is a natural question to address, for it provides the relation between the characteristic wavelength of the bifurcation mode on one hand and the corresponding layer parameters, such as stresses, tangent moduli and densities, on the other. A theoretical framework for the analysis of bifurcation instabilities has been established for elastic solids by KoITER (1945) and for rate independent elastoplastic solids by HILL (1958) and the same subjects have been reviewed comprehensively by BUDIANSKY (1974) and HUTCHINSON (1974).

For a general formulation of the type of problem considered here, the governing equations will be stated first in a full Lagrangian form. Accordingly, the material points of a body of volume $V$ and bounding surface $\partial V$ in the undeformed configuration are identified by their initial Cartesian coordinates $X_{i}$, while the components of the displacement vector are denoted by $u_{i}$. A superimposed dot-such as on $\dot{u}_{i}-$ designates the derivative of a field variable with respect to some monotonically increasing time-like parameter that traces the evolution of stress and deformation states during a loading process. The comma notation $u_{i, j}$ is used to denote partial differentiation with respect to $X_{j}$. Einstein's summation convention over repeated indices is adopted throughout this work, with Latin indices ranging from 1 to 2 in plane strain problems and from 1 to 3 in more general three-dimensional formulations.

The prebifurcation state of the body or material region that will be considered is characterized by a planar interface between the two layers and a horizontally uniform stress state. This fundamental solution can be completely specified as a function of a monotonically increasing scalar quantity $\Lambda$, the load parameter. At every stage of the deformation process one may seek the incremental response of the solid layer--in terms of the rates $\dot{\pi}_{i j}$ and $\dot{u}_{i}$ of the first Piola-Kirchhoff stress $\pi_{i j}$ and displacement vector $u_{i}$ in the interior of the layer - for a given $\dot{\Lambda}$ that specifics a boundary traction increment $\dot{T}_{i}$ or boundary displacement increment $\dot{u}_{i}$.

The equation of equilibrium for the solid, written in its variational (or weak) form, is

$$
\int_{V} \pi_{j i} \delta F_{i j} \mathrm{~d} V=\int_{V} \rho b_{i} \delta u_{i} \mathrm{~d} V+\int_{\hat{i} V} T_{i} \delta u_{i} \mathrm{~d} A, \quad F_{i j}=\delta_{i j}+u_{i, j}
$$

where $F_{i j}$ denote the components of the deformation gradient tensor, $\rho$ the density of the solid per unit reference volume and $b_{i}$ the components of the acceleration of gravity. Assuming that $\rho$ and $b_{i}$ are time-independent constants for each layer, the condition of continuing equilibrium, i.e. the rate-form of eqn (1), becomes

$$
\int_{V} \dot{\pi}_{j i} \delta F_{i j} \mathrm{~d} V=\int_{\partial V} \dot{T}_{i} \delta u_{i} \mathrm{~d} A
$$

Suppose now that at some value of $\Lambda$ a bifurcation in the deformational response of the solid becomes posssible, so that for a given increment $\dot{\Lambda}$ there exist two different solutions $\dot{\pi}_{i j}^{\alpha}, \dot{u}_{i}^{\alpha}$ and $\dot{\pi}_{i j}^{\beta}, \dot{u}_{i}^{\beta}$. Let $\Delta(\cdot) \equiv(\cdot)^{\beta}-(\cdot)^{\alpha}$ denote the difference between two such solutions for any ficld quantity $(\cdot)$. At every boundary point either $\Delta \dot{T}_{i}=0$, if 
tractions are prescribed, or $\delta \dot{u}_{i}=0$, if displacements are prescribed. The difference between any two incremental equilibrium solutions $\alpha$ and $\beta$ to eqn (2) must therefore satisfy

$$
\int_{V} \Delta \dot{\pi}_{j i} \delta F_{i j} \mathrm{~d} V=\int_{i V} \Delta \dot{T}_{i} \delta u_{i} \mathrm{~d} A=0
$$

An integration by parts now furnishes both the pointwise incremental cquilibrium or Euler-Lagrange equation, as well as the corresponding boundary and interface conditions. The latter are fully determined only by an additional adherence condition, which will be assumed to hold for the material on either side of the overburden/ substratum boundary. One obtains

$$
\begin{aligned}
\left(\Delta \dot{\pi}_{i j}\right)_{, i} & =0 & & \text { (at interior points of } V), \\
\llbracket \Delta \dot{\pi}_{i j} N_{i} \rrbracket & =0, \quad \llbracket \Delta \dot{u}_{i} \rrbracket=0 & & \text { (at the interface), }
\end{aligned}
$$

where $N_{i}$ are the components of the unit normal to the material interface and the customary bracket notation $[. .$.$] is employed to denote a jump in a field quantity$ across that boundary. The second jump condition expresses the assumption of perfect bonding of the two materials. To complete the formulation, further conditions must be imposed on $\Delta \dot{u}_{i}$ and $\Delta \dot{F}_{i j}$ at infinite distances from the interface; these will be considered later in the context of specific applications.

The rate-independent materials that are to be considered will be assumed to obey the following constitutive equations :

$$
\begin{aligned}
& \dot{\pi}_{j i}=L_{i j k i} \dot{u}_{l, k} \quad \text { (compressible solid), } \\
& \dot{\pi}_{j i}=L_{i j k l} \dot{u}_{l, k}-\dot{p}\left(\dot{\delta}_{j i}+u_{j, i}\right)^{-1}, \quad \operatorname{det}\left(\delta_{i j}+u_{i, j}\right)=1 \quad \text { (incompressible solid), }
\end{aligned}
$$

where the second equation involves the pressure increment $\dot{p}$. The components $L_{i j k}$ of the incremental modulus tensor generally depend on the current stress state as well as on the stress path from the reference to the current state. Having selected one or the other form of (5), one may now seek a solution to the incremental boundary value problem, such that $\dot{\pi}_{i j}$ and $\dot{u}_{i}$ are found in the interior of $V$ for a given increment $\dot{\Lambda}$ or corresponding increments in $\dot{T}_{i}$ and $\dot{u}_{i}$ along $\partial V$.

Equations (4) and (5) provide a basic set of equations for studying the bifurcation problem that is associated with the stability of the overburden/substratum interface. They are written in terms of a fixed reference configuration, corresponding to a full Lagrangian formulation. For problems, such as the present one, that are characterized by simple prebifurcation states, it is often convenient to select the current configuration as the reference configuration and accordingly, to take $u_{i}=0, F_{i j}=\delta_{i j}$, but $\dot{u}_{i} \neq 0$, $\dot{F}_{i j} \neq 0$. This updated Lagrangian formulation will be used in this paper, its advantage lying in the use of Cauchy stress and in the fact that the mode shapes are measured in the current configuration at the onset of the bifurcation.

Next, in considering the stability of an initially flat interface, it will suffice to investigate the behaviour of the system when subjected at time $t=0$ to a perturbation 
of small amplitude $\varepsilon$ but arbitrary wavelength about the equilibrium state of interest. This is achieved by studying the solution of the linearized dynamical equations for the system. The starting point for this analysis is the weak form of the system's equations of motion

$$
\int_{V} \pi_{j i} \delta F_{i j} \mathrm{~d} V=\int_{V} \rho\left(b_{i}-\ddot{u}_{i}\right) \delta u_{i} \mathrm{~d} V+\int_{\partial V} T_{i} \delta u_{i} \mathrm{~d} A, \quad u_{i}, \dot{u}_{i} \text { given at } t=0 .
$$

All field quantities for the perturbed system can be written as a sum of their corresponding fundamental (unperturbed) values, which are denoted by a superscript $\left({ }^{\circ}\right)$, plus a term that depends on the amplitude $\varepsilon$ of the initial disturbance. By expanding these field quantities with respect to the initial disturbance amplitude, one has for the first Piola-Kirchhoff stress, the displacement and pressure fields

$$
\pi_{i j}=\pi_{i j}^{0}+\varepsilon \tilde{\pi}_{i j}+\mathrm{O}\left(\varepsilon^{2}\right), \quad u_{i}=u_{i}^{0}+\varepsilon \tilde{u}_{i}+\mathrm{O}\left(\varepsilon^{2}\right), \quad p=p^{0}+\varepsilon \tilde{p}+\mathrm{O}\left(\varepsilon^{2}\right) .
$$

It is worth mentioning at this point that all $O(1)$ field quantities associated with the fundamental solution are functions of $\Lambda$ and are independent of time, unlike all the $\mathrm{O}\left(\varepsilon^{\mathrm{p}}\right)(p \geqslant 1)$ dependent terms in the perturbation which do depend on time.

A straightforward linearization of the equations of motion obtained by introducing (7) into (6) and by keeping only the terms of $O(\varepsilon)$ gives the linearized equations of motion

$$
\begin{aligned}
\left(\tilde{\pi}_{i j}\right)_{, i} & =\rho \ddot{\tilde{u}}_{i} \quad & & \text { (at interior points of } V \text { ), } \\
\llbracket \tilde{\pi}_{i j} N_{i} \rrbracket & =0, \quad \llbracket \tilde{u}_{i} \rrbracket=0 & & \text { (at the material interface) } .
\end{aligned}
$$

Moreover, the same linearization procedure applied to the constitutive equation (5) yields

$$
\begin{array}{ll}
\dot{\tilde{\pi}}_{j i}=L_{i j k l} \dot{\tilde{u}}_{l, k} & \text { (compressible solid) }, \\
\dot{\tilde{\pi}}_{j i}=L_{i j k i} \dot{\tilde{u}}_{l, k}-\dot{\tilde{p}} \delta_{j i}, \quad \dot{\tilde{u}}_{i, i}=1 & \text { (incompressible solid) } .
\end{array}
$$

The above two sets of equations (8) and (9), complemented by the initial conditions in $\tilde{u}_{i}$ and $\dot{\tilde{u}}_{i}$ which are given at $t=0$ completely specify the $O(\varepsilon)$ term in the expansion of the perturbed system.

Following the standard assumption adopted in linearized stability analysis, it is assumed that the omitted $O\left(\varepsilon^{2}\right)$ terms are negligible over the time interval of interest and that the first order terms adequately characterize the motion of the system. Since the coefficients in the system of linearized equations of motion do not depend on time, the solution to (8) and (9), subject to the aforementioned initial conditions of given displacement and velocity, takes the form

$$
\left.\begin{array}{l}
\tilde{p}\left(x_{1}, x_{2}, x_{3}, t\right) \\
\tilde{u}_{i}\left(x_{1}, x_{2}, x_{3}, t\right) \\
\tilde{\pi}_{i j}\left(x_{1}, x_{2}, x_{3}, t\right)
\end{array}\right\}=\mathrm{e}^{i \zeta t}\left\{\begin{array}{l}
\hat{p}\left(x_{1}, x_{2}, x_{3}\right) \\
\hat{u}_{i}\left(x_{1}, x_{2}, x_{3}\right) . \\
\hat{\pi}_{i j}\left(x_{1}, x_{2}, x_{3}\right)
\end{array}\right.
$$

Upon introducing this in (8) onc arrives at the following eigenvalue problem 


$$
\begin{aligned}
\hat{\pi}_{i j, i}+\rho \xi^{2} \hat{u}_{i} & =0 & & \text { (at interior points of } V \text { ), } \\
\llbracket \hat{\pi}_{i j} N_{i} \rrbracket & =0, \quad \llbracket \hat{u}_{i} \rrbracket=0 & & \text { (at the interface), }
\end{aligned}
$$

where the relation between $\hat{\pi}_{i j}, \hat{u}_{i}$ and $\hat{p}$ can be easily found following a substitution of (10) into (9)

$$
\begin{array}{ll}
\hat{\pi}_{j i}=L_{i j k l} \hat{u}_{i, k} & \text { (compressible solid), } \\
\hat{\pi}_{j i}=L_{i j k i} \hat{u}_{l, k}-\hat{p} \delta_{j i}, \quad \hat{u}_{i, i}=1 & \text { (incompressible solid). }
\end{array}
$$

Notice that $\xi^{2}$ is the linear eigenvalue of the above system of equations (11), (12). A more recognizable form of the eigenvalue problem, which is the weak formulation of the above equations, is obtained by introducing (12) into (11), multiplying the resulting equation by $\delta u_{i}$ and subsequently integrating over the volume $V$ of the solid

$$
\int_{V} L_{i j / k} \hat{u}_{k, l} \delta u_{i, j} \mathrm{~d} V=\xi^{2} \int_{V} \rho \hat{u}_{i} \delta u_{i} \mathrm{~d} V .
$$

When the incremental moduli possess the symmetry property $L_{i j l k}=L_{l k i j}$ as will be in the case of applications considered here, all eigenvalues $\xi^{2}$ are real. Consequently if the minimum eigenvalue $\xi_{m}^{2}>0$, it follows that the solution (10) must remain bounded in time so that the interface is stable (recall that no dissipation mechanism is included in the system; the presence of dissipation--inevitable in reality--will result in the decay of the solution's amplitude). For $\xi_{m}^{2}<0(10)$ will permit solutions that increase without bound, implying that the system is unstable. It is also seen that if $\xi^{2}=0$ for a certain perturbation, then eqns (11), (12) and (4), (5) coincide and the eigenmode $\hat{u}_{i}$ becomes the bifurcation eigenmode $\left(\hat{u}_{i} \equiv \Delta \dot{u}_{i}\right)$.

\section{Model Formulation, Bifurcation Criterion and Linearized Stability}

In the interest of analytical simplicity, only the plane strain bifurcation and stability problem for the interface between two solid half-spaces will be analysed here. The constitutive description will be kept as simple as possible in terms of a rate and pressure insensitive, incompressible and orthotropic solid that has one axis of orthotropy oriented parallel to the interface. As was shown by Bır (1965), the constitutive equation of such a solid may then be stated as follows:

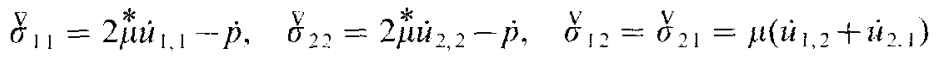

in terms of the objective Jaumann rate $\sigma_{i j}$ of the Cauchy stress, the hydrostatic pressure $p$ and the two incremental moduli $\mu$ and $\mu$ that characterize the matcrial. The relation between the rate of the first Piola-Kirchhoff stress and the Jaumann rate of the Cauchy stress for an incompressible material is [see HiLl and Hutchinson (1975)]

$$
\dot{\pi}_{i j}=\sigma_{i j}-\frac{1}{2} \sigma_{i k}\left(\dot{u}_{k, j}-\dot{u}_{j, k}\right)-\frac{1}{2}\left(\dot{u}_{i, k}+\dot{u}_{k, i}\right) \sigma_{k j} .
$$

Equations (14) and (15) therefore give 


$$
\begin{aligned}
& \dot{\pi}_{11}=\left(2 \mu-\sigma_{11}\right) \dot{u}_{1,1}-\dot{p}, \\
& \dot{\pi}_{22}=\left(2 \mu-\sigma_{22}\right) \dot{u}_{2,2}-\dot{p}, \\
& \dot{\pi}_{12}=\left(\mu-\frac{\sigma_{22}-\sigma_{11}}{2}\right) \dot{u}_{2,1}+\left(\mu-\frac{\sigma_{11}+\sigma_{22}}{2}\right) \dot{u}_{1,2}, \\
& \dot{\pi}_{21}=\left(\mu+\frac{\sigma_{22}-\sigma_{11}}{2}\right) \dot{u}_{1,2}+\left(\mu-\frac{\sigma_{11}+\sigma_{22}}{2}\right) \dot{u}_{2,1} .
\end{aligned}
$$

In deriving (16) from (14) and (15), the fact has been used that the prebifurcation stress state and the material are orthotropic with respect to the same axes, so that $\sigma_{12}=0$ [see Biot (1965) for further details]. Moreover, the condition of incompressibility $\operatorname{det}\left(F_{i j}\right)=1$, when expressed in the updated I agrangian formulation, requires that

$$
\dot{u}_{1,1}+\dot{u}_{2.2}=0 \text {. }
$$

One can easily verify that the incremental constitutive equation (16) is of the general form $(5)_{2}$, in agreement with the assumption made in the previous section.

A tacit assumption underlying the subsequent use of (14) is that it is applicable to the initial constitutive response on the bifurcated equilibiium branch as well (i.e. the unloading that might occur in the postbifurcation solution is not considered in formulating the bifurcation problem). Indeed, as was shown by HiLL (1958), providing the principal solution satisfies the condition of plastic loading everywhere (as is true for the present problem), the tangent moduli $\mu$ and $\stackrel{*}{\mu}$ of the plastic loading response can be safely employed in the bifurcation analysis, since the resulting critical load is less than or equal to the actual bifurcation load. Working on the postbifurcation response of elastoplastic solids, HuTCHInson (1974) has further shown that the use of the tangent moduli $\mu$ and $\stackrel{*}{\mu}$ of the plastic loading response gives the correct bifurcation load, provided that the prebifurcation solution satisfies plastic loading everywhere. Consideration of unloading is required for the calculation of the postbifurcated solution.

Consider now two incompressible orthotropic half-spaces of densities $\rho^{a}$ and $\rho^{b}$, respectively, perfectly bonded along the interface $x_{2}=0$ as shown in Fig. 1. The axes of orthotropy of each half-space are aligned with the coordinate axes $x_{1}, x_{2}$ and their constitutive response is given by (14). The prebifurcation stress state is also or thotropic with respect to the same axes. Gravity acts in the negative $x_{2}$ direction so that $b_{1}=0$, $b_{2}=-g$ in (1). Although the incremental moduli $\mu$ and $\stackrel{*}{\mu}$ depend on the history of deformation, for pressure insensitive materials and assuming that proportional loading is a reasonable approximation of the loading history, these moduli depend on $\left|\sigma_{22}-\sigma_{11}\right|$ only, so that

$$
\mu=\mu(\tau), \quad \stackrel{*}{\mu}=\stackrel{*}{\mu}(\tau) ; \quad \tau \equiv\left(\sigma_{22}-\sigma_{11}\right) / 2, \quad \sigma \equiv\left(\sigma_{11}+\sigma_{22}\right) / 2
$$

Henceforth omission of the subscript identifying a layer in an equation will imply that the relevant quantities are either defined for both layers or else are to be associated with one particular layer that will be recognized from the context. 


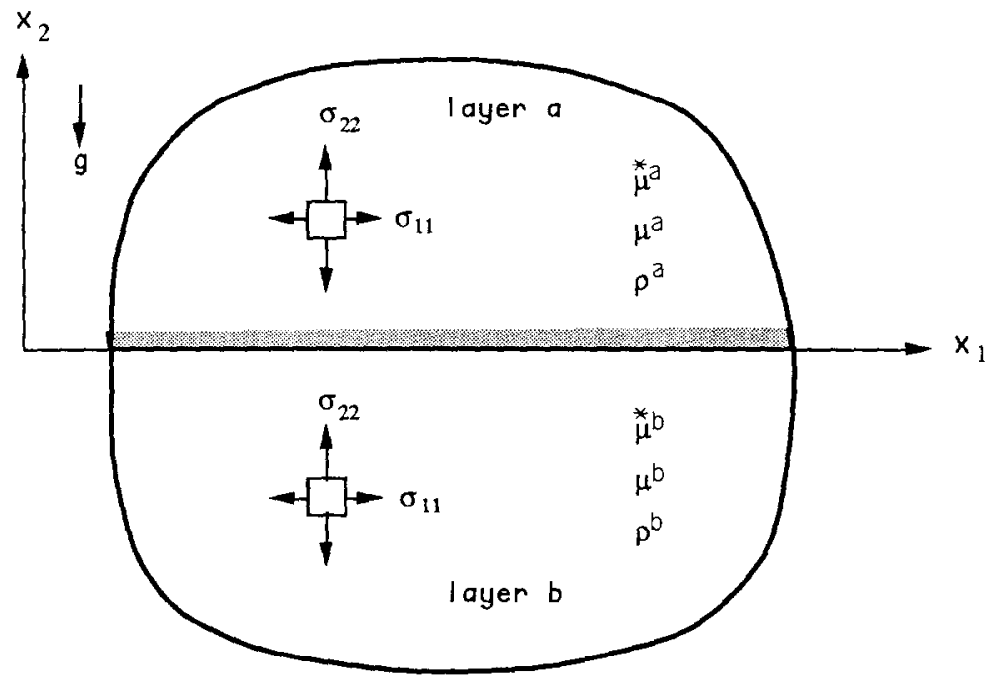

FlG. I. Coherent interface $x_{2}=0$ between two semi-infinite media in a gravitational field $g$.

In order to keep the subsequent analysis as simple as possible, the following prebifurcation stress state is assumed

$$
\sigma_{11}=(1-2 k) \rho g x_{2}+\sigma_{1}, \quad \sigma_{22}=\rho g x_{2}+\sigma_{2}, \quad \sigma_{12}=0 .
$$

The constant $k$ as well as the stresses $\sigma_{1}$ and $\sigma_{2}$ at the interface will in general take on different values in each half-space, except for the condition $\sigma_{2}^{a}=\sigma_{2}^{b}$ that is demanded by equilibrium across the interface. The parameter $k$ plays an important role in that it determines the gradient in the stress deviator within each layer according to $\mathrm{d} \tau / \mathrm{d} x_{2}=k \rho g$, as may be seen from (18) and (19). Since $\tau$ governs the constitutive response of the material, its gradient must have a controlling influence on any bifurcation instability. Hence, if $k=0$, there will be no stress gradient effect on the bifurcation behaviour. As discussed in the previous section, an updated Lagrangian formulation of the field equations will be used for which the reference configuration coincides with the current configuration at the onset of bifurcation when $u_{i}=0$ while $\dot{u}_{i} \neq 0$. The condition of incompressibility (17) ensures the existence of a potential function $\psi$, satisfying

$$
\Delta \dot{u}_{1}=\psi_{.2}, \quad \Delta \dot{u}_{2}=-\psi_{.1}
$$

When (14) and (20) are introduced into the incremental equilibrium equations (4) one obtains

$$
\begin{aligned}
& {\left[\left(2 \mu-\sigma_{11}\right) \psi_{.12}-\Delta \dot{p}\right]_{.1}+\left[(\mu+\tau) \psi_{.22}-(\mu-\sigma) \psi_{, 11}\right]_{, 2}=0} \\
& {\left[\left(2 \mu-\sigma_{22}\right) \psi_{.12}+\Delta \dot{p}\right]_{, 2}+\left[(\mu-\tau) \psi_{.11}-(\mu-\sigma) \psi_{.22}\right]_{, 1}=0 .}
\end{aligned}
$$


The corresponding jump conditions across the interface $x_{2}=0$, with unit normal $n_{i}=\delta_{i 2}$, are

$$
\begin{aligned}
& \llbracket \psi, 1 \rrbracket=0, \\
& \llbracket \psi_{, 2} \rrbracket=0, \\
& \llbracket(\mu+\tau) \psi_{, 22}-(\mu-\sigma) \psi_{, 11} \rrbracket=0, \\
& \llbracket\left(2 \mu-\sigma_{22}\right) \psi_{, 12}+\Delta \dot{p} \rrbracket=0 .
\end{aligned}
$$

The first two of these relations are a consequence of the continuity requirement (4) for the displacement rate, while the last two express the traction rate continuity (4) ${ }_{2}$. In addition to (21) and (22) one requires conditions at infinity for $\psi$. The validity of the present double-half-space model as an approximation for the more realistic problem of the instability of a finite-thickness layer resting on a half-space depends upon an insensitivity of the interfacial response to the exact free-surface boundary conditions. Accordingly only those eigenmodes $\Delta \dot{u}_{i}$ that decay away from the interface will be studied; from (20) the required condition is that $\psi_{, 1}, \psi_{, 2} \rightarrow 0$ as $\left|x_{2}\right| \rightarrow \infty$.

Next, one eliminates the pressure from the governing equations (21) and (22) to obtain

$$
\left[(\mu-\tau) \psi_{.11}-(\mu-\sigma) \psi_{.22}\right]_{.11}+2\left[(2 \mu-\sigma) \psi_{.12}\right]_{.12}+\left[(\mu+\tau) \psi_{.22}-(\mu-\sigma) \psi_{, 11}\right]_{, 22}=0,
$$

and the interface conditions

$$
\begin{aligned}
& \llbracket \psi_{, 1} \rrbracket=0, \\
& \llbracket \psi_{, 2} \rrbracket=0, \\
& \llbracket(\mu+\tau)\left(\psi_{, 22}-\psi_{, 11}\right) \rrbracket=0, \\
& \llbracket 2\left[(2 \mu+\tau) \psi_{.12}\right]_{, 1}+\left[(\mu+\tau)\left(\psi_{, 22}-\psi_{.11}\right)\right]_{.2}+\sigma_{22,2} \psi_{, 11} \rrbracket=0
\end{aligned}
$$

The coefficients in eqn (23) and the boundary conditions (24) are independent of $x_{1}$ (the aforementioned boundary value problem being translationally invariant with respect to $\left.x_{1}\right)$. This suggests the use of the Fourier transform $\tilde{\psi}=\mathscr{F}\left[\psi\left(x_{1}, x_{2}\right), x_{1} \rightarrow\right.$ $\omega]$ for obtaining an ordinary differential equation in $x_{2}$. Also, the new variable $y \equiv-\omega x_{2}$ will henceforth be used. Consequently, and in view of equations (18) and (19), the governing equation (23) assumes the form

$$
\begin{aligned}
(1-\tau / \mu) \tilde{\psi}+2 k \frac{\rho g}{\mu \omega}\left(2 \frac{\mathrm{d} \mu}{\mathrm{d} \tau}-\frac{\mathrm{d} \mu}{\mathrm{d} \tau}\right) \frac{\mathrm{d} \tilde{\psi}}{\mathrm{d} y} & -\left[2(2 \mu / \mu-1)-k^{2}\left(\frac{\rho g}{\mu \omega}\right)^{2} \mu \frac{\mathrm{d}^{2} \mu}{\mathrm{d} \tau^{2}}\right] \frac{\mathrm{d}^{2} \tilde{\psi}}{\mathrm{d} y^{2}} \\
& -2 k \frac{\rho g}{\mu \omega}\left(\frac{\mathrm{d} \mu}{\mathrm{d} \tau}+1\right) \frac{\mathrm{d}^{3} \tilde{\psi}}{\mathrm{d} y^{3}}+(1+\tau / \mu) \frac{\mathrm{d}^{4} \tilde{\psi}}{\mathrm{d} y^{4}}=0 .
\end{aligned}
$$

The corresponding interface conditions at $y=0$ are 
$\llbracket \tilde{\psi} \rrbracket=0$,

$\left[\begin{array}{l}\mathrm{d} \tilde{\psi} \\ \mathrm{d} y\end{array}\right]=0$

$\left[\mu(1+\tau / \mu)\left(\frac{\mathrm{d}^{2} \tilde{\psi}}{\mathrm{d} y^{2}}+\tilde{\psi}\right)\right]=0$

$\left[\mu\left[(4 \mu / \mu-1+\tau / \mu) \frac{\mathrm{d} \tilde{\psi}}{\mathrm{d} y}-(1+\tau / \mu) \frac{\mathrm{d}^{3} \tilde{\psi}}{\mathrm{d} y^{3}}+k \frac{\rho g}{\mu \omega}\left(\frac{\mathrm{d} \mu}{\mathrm{d} \tau}+1\right)\left(\frac{\mathrm{d}^{2} \tilde{\psi}}{\mathrm{d} y^{2}}+\tilde{\psi}\right)-\frac{\rho g}{\mu \omega} \tilde{\psi}\right] \rrbracket=0\right.$.

Equations (25) and (26) are complemented by the condition $\tilde{\psi} \rightarrow 0$ as $|y| \rightarrow \infty$. The amplitude of the eigenmode is expected to decay exponentially away from the interface. It will be assumed that the coefficients appearing in (25) and (26) do not vary significantly within the decay distance of the eigenmode so that they may be approximated by their values at $y=0$. The solution to (25) of interest is then given by

$\tilde{\psi}(\omega, y)=A_{1}(\omega) \mathrm{e}^{-y^{y}}+A_{2}(\omega) \mathrm{e}^{z_{2} y}, \quad\left\{\begin{array}{lll}\operatorname{Re}\left(z_{\gamma}\right)<0, & \text { if } y>0, & \text { (half-space } b), \\ \operatorname{Re}\left(z_{\gamma}\right)>0, & \text { if } y<0, & \text { (half-space } a \text { ), }\end{array}\right.$

taking $\omega>0$. Here $z_{1}, z_{2}\left(z_{1} \neq z_{2}\right)$ are roots of the fourth order polynomial

$$
\begin{array}{r}
1-\tau / \mu+2 k \frac{\rho g}{\mu \omega}\left(2 \frac{\mathrm{d}^{*} \mu}{\mathrm{d} \tau}-\frac{\mathrm{d} \mu}{\mathrm{d} \tau}\right) z-\left[2\left(2 \mu^{*} / \mu-1\right)-k^{2}\left(\frac{\rho g}{\mu \omega}\right)^{2} \mu \frac{\mathrm{d}^{2} \mu}{\mathrm{d} \tau^{2}}\right] z^{2} \\
-2 k \frac{\rho g}{\mu \omega}\left(\frac{\mathrm{d} \mu}{\mathrm{d} \tau}+1\right) z^{3}+(1+\tau / \mu) z^{4}=0 .
\end{array}
$$

Since the admissible modes are required to decay to zero away from the interface, it is the range of parameters for which the above equation has two roots with a positive (negative) real part in half-space $a(b)$ that is of interest, for eqn (23) will be elliptic in this case. Notice also, that substitution of $z=i\left(n_{1} / n_{2}\right)$ in (20) yields the characteristic equation of $(23),\left(n_{1}, n_{2}\right)$ being the unit normal to the characteristic lines. Equation (23) is elliptic in character, if it possesses no real characteristics. The loss of ellipticity of (23)--for certain values of its coefficients - entails the possibility of discontinuous solutions, that is strain discontinuities in the solid in the form of shear bands. However, as discussed by HiLl and HuTCHINSON (1975), Young (1976) and RICE (1976), such discontinuities will always appear at higher stress levels than a bifurcation with eigenmodes that are varying smoothly in space. The bifurcation is thus expected to occur, when at the interface the material parameters lie in the elliptic range.

Introducing (27) into the boundary conditions (26), one has 


$$
\begin{aligned}
& \llbracket A_{1}+A_{2} \rrbracket=0, \\
& \left.\llbracket z_{1} A_{1}+z_{2} A_{2}\right]=0, \\
& \llbracket \mu(1+\tau / \mu)\left(z_{1}^{2} A_{1}+z_{2}^{2} A_{2}+A_{1}+A_{2}\right) \rrbracket=0, \\
& \llbracket\left[(4 \mu / \mu-1+\tau / \mu)\left(z_{1} A_{1}+z_{2} A_{2}\right)-(1+\tau / \mu)\left(z_{1}^{3} A_{1}+z_{2}^{3} A_{2}\right)\right. \\
& \left.\qquad+k \frac{\rho g}{\mu \omega}\left(\frac{\mathrm{d} \mu}{\mathrm{d} \tau}+1\right)\left(z_{1}^{2} A_{1}+z_{2}^{2} A_{2}+A_{1}+A_{2}\right)-\frac{\rho g}{\mu \omega}\left(A_{1}+A_{2}\right)\right] \rrbracket=0 .
\end{aligned}
$$

The continuity of $A_{1}+A_{2}$ and $z_{1} A_{1}+z_{2} A_{2}$ across the interface enables one to make use of the following convenient notation

$$
A_{1}^{u}+A_{2}^{u}=A_{1}^{b}+A_{2}^{u} \equiv \Sigma_{1}, \quad z_{1}^{u} A_{1}^{u}+z_{2}^{u} A_{2}^{u}=z_{1}^{b} A_{1}^{b}+z_{2}^{b} A_{2}^{b} \equiv \Sigma_{2} .
$$

Moreover, one has the identities

$$
\begin{aligned}
& z_{1}^{2} A_{1}+z_{2}^{2} A_{2}=\left(z_{1}+z_{2}\right) \Sigma_{2}-z_{1} z_{2} \Sigma_{1} \\
& z_{1}^{3} A_{1}+z_{2}^{3} A_{2}=\left(z_{1}^{2}+z_{1} z_{2}+z_{2}^{2}\right) \Sigma_{2}-z_{1} z_{2}\left(z_{1}+z_{2}\right) \Sigma_{1},
\end{aligned}
$$

in terms of which (29) can be written

$$
C_{12} \Sigma_{2}+C_{11} \Sigma_{1}=0, \quad C_{22} \Sigma_{2}+C_{21} \Sigma_{1}=0
$$

with

$$
\begin{aligned}
& C_{12} \equiv \llbracket \mu(1+\tau / \mu)\left(z_{1}+z_{2}\right) \rrbracket, \\
& C_{11} \equiv \llbracket \mu(1+\tau / \mu)\left(1-z_{1} z_{2}\right) \rrbracket, \\
& C_{22} \equiv \llbracket\left[\mu\left[\left(4 \mu^{*} / \mu-1+\tau / \mu\right)-(1+\tau / \mu)\left(z_{1}^{2}+z_{1} z_{2}+z_{2}^{2}\right)+k \frac{\rho g}{\mu \omega}\left(\frac{\mathrm{d} \mu}{\mathrm{d} \tau}+1\right)\left(z_{1}+z_{2}\right)\right] \rrbracket,\right. \\
& C_{21} \equiv \llbracket\left[\mu\left[(1+\tau / \mu) z_{1} z_{2}\left(z_{1}+z_{2}\right)+k \frac{\rho g}{\mu \omega}\left(\frac{\mathrm{d} \mu}{\mathrm{d} \tau}+1\right)\left(1-z_{1} z_{2}\right)-\frac{\rho g}{\mu \omega}\right] \rrbracket .\right.
\end{aligned}
$$

One trivial solution of the above system is $\Sigma_{1}=\Sigma_{2}=0$ which, on account of (27), implies that $\widetilde{\psi}=\psi=0$, thus excluding bifurcation. A bifurcation becomes possible only when a non-zero solution for $\tilde{\psi}$ can be found, that is if

$$
C_{12} C_{21}-C_{11} C_{22}=0 \text {. }
$$

In this bifurcation condition the matrix coefficients $C_{i j}$ are given by (32) while the $z_{\gamma}$ are appropriate roots of the fourth order polynomial in (28). Condition (33) can be considered as an implicit equation in $\omega$, since the $C_{i j}$ and $z_{\gamma}$ are functions of $\omega$. The critical wavenumber $\omega_{c}$ that will permit the first appearance of a bifurcation at the interface of the two half-spaces can thus be found as a function of the densities, the stresses and the incremental moduli as well as their derivatives, all evaluated at the interface. Needless to say that, in general, $\omega_{c}$ will only be obtainable by solving (33) numerically.

Attention is now focused on determining the stability of an initially flat interface, based on the sign criterion for the minimum eigenvalue of $(11),(12)$ as discussed in 
the previous section. The perturbation $\hat{u}_{i}$ in the displacement field again satisfies the condition of incompressibility $\hat{u}_{1,1}+\hat{u}_{2,2}=0$ which is satisfied identically by a stream function $\psi$ such that $\hat{u}_{1}=\psi_{, 2}$ and $\hat{u}_{2}=-\psi_{\text {.1 }}$. Upon making use of this representation in $(9)_{1}$, while substituting for $\hat{\pi}_{i j}$ a constitutive relation of the form (15), one arrives at

$$
\begin{aligned}
& {\left[\left(2 \mu-\sigma_{11}\right) \psi_{, 12}-\hat{p}\right]_{, 1}+\left[(\mu+\tau) \psi_{.22}-(\mu-\sigma) \psi_{, 11}\right]_{, 2}+\rho \xi^{2} \psi_{, 2}=0} \\
& {\left[\left(2 \mu-\sigma_{22}\right) \psi_{.12}+\hat{p}\right]_{, 2}+\left[(\mu-\tau) \psi_{, 11}-(\mu-\sigma) \psi_{.22}\right]_{.1}-\rho \xi^{2} \psi_{.1}=0}
\end{aligned}
$$

and, after elimination of $\hat{p}$,

$$
\begin{array}{r}
{\left[(\mu-\tau) \psi_{, 11}-(\mu-\sigma) \psi_{, 22}\right]_{, 11}+2\left[\left(2{ }^{*}-\sigma\right) \psi_{.12}\right]_{.12}+\left[(\mu+\tau) \psi_{.22}-(\mu-\sigma) \psi_{, 11}\right]_{.22}} \\
+\rho \xi^{2}\left(\psi_{, 11}+\psi_{.22}\right)=0
\end{array}
$$

The relations pertaining to the interface are obtained through steps that parallel the derivation of (24), resulting in

$$
\begin{aligned}
& \llbracket \psi_{, 1} \|=0, \\
& \llbracket \psi_{, 2} \rrbracket=0, \\
& \llbracket(\mu+\tau)\left(\psi_{.22}-\psi_{, 11}\right) \rrbracket=0, \\
& \llbracket\left[(2 \stackrel{*}{\mu}+\tau) \psi_{.12}\right]_{, 1}+\left[(\mu+\tau)\left(\psi_{, 22}-\psi_{, 11}\right)\right]_{, 2}+\sigma_{22,2} \psi_{, 11}+\rho \xi^{2} \psi, 2 \rrbracket=0 .
\end{aligned}
$$

A Fourier transformation applied to (34) and (35) yields

$$
\begin{aligned}
& \left(1-\tau / \mu-\frac{\rho \xi^{2}}{\mu \omega^{2}}\right) \tilde{\psi}+2 k \frac{\rho g}{\mu \omega}\left(2 \frac{\mathrm{d} \mu}{\mathrm{d} \tau}-\frac{\mathrm{d} \mu}{\mathrm{d} \tau}\right) \frac{\mathrm{d} \tilde{\psi}}{\mathrm{d} y}-[2(2 \mu / \mu-1) \\
& \left.\quad-k^{2}\left(\frac{\rho g}{\mu \omega}\right)^{2} \mu \frac{\mathrm{d}^{2} \mu}{\mathrm{d} \tau^{2}}-\frac{\rho \xi^{2}}{\mu \omega^{2}}\right] \frac{\mathrm{d}^{2} \tilde{\psi}}{\mathrm{d} y^{2}}-2 k \frac{\rho g}{\mu \omega}\left(\frac{\mathrm{d} \mu}{\mathrm{d} \tau}+1\right) \frac{\mathrm{d}^{3} \tilde{\psi}}{\mathrm{d} y^{3}}+(1+\tau / \mu) \frac{\mathrm{d}^{4} \tilde{\psi}}{\mathrm{d} y^{4}}=0,
\end{aligned}
$$

in complete correspondence with (25), recalling that $y \equiv-\omega x_{2}$. The corresponding interface conditions at $y=0$ are

$\llbracket \widetilde{\psi} \rrbracket=0$,

$\llbracket \frac{\mathrm{d} \tilde{\psi}}{\mathrm{d} y} \rrbracket=0$

$\left[\mu(1+\tau / \mu)\left(\frac{\mathrm{d}^{2} \tilde{\psi}}{\mathrm{d} y^{2}}+\tilde{\psi}\right)\right]=0$,

$\left[\mu\left[\left(4 * / \mu-1+\tau / \mu-\frac{\rho \xi^{2}}{\mu \omega^{2}}\right) \frac{\mathrm{d} \tilde{\psi}}{\mathrm{d} y}-(1+\tau / \mu) \frac{\mathrm{d}^{3} \tilde{\psi}}{\mathrm{d} y^{3}}\right.\right.$

$$
\left.\left.+k \frac{\rho g}{\mu \omega}\left(\frac{\mathrm{d} \mu}{\mathrm{d} \tau}+1\right)\left(\frac{\mathrm{d}^{2} \tilde{\psi}}{\mathrm{d} y^{2}}+\tilde{\psi}\right)-\frac{\rho g}{\mu(\alpha)} \tilde{\psi}\right]\right]=0 .
$$


Since the amplitude of the perturbation is expected to decay exponentially away from the interface, it will be assumed that $\tilde{\psi} \rightarrow 0$ as $|y| \rightarrow \infty$ and that the coefficients appearing in (37) and (38) may be approximated by their values at $y=0$. The solution to (37) of interest is therefore again of the form (27), $z_{1}$ and $z_{2}$ being the roots with positive (negative) real part in halfspace $a(b)$ of the fourth order polynomial

$$
\begin{aligned}
1-\tau / \mu-\frac{\rho \xi^{2}}{\mu \omega^{2}}+2 k \frac{\rho g}{\mu \omega}\left(2 \frac{\mathrm{d} \mu}{\mathrm{d} \tau}-\frac{\mathrm{d} \mu}{\mathrm{d} \tau}\right) z- & {\left[2(2 \mu / \mu-1)-k^{2}\left(\frac{\rho g}{\mu \omega}\right)^{2} \mu \frac{\mathrm{d}^{2} \mu}{\mathrm{d} \tau^{2}}-\frac{\rho \xi^{2}}{\mu \omega^{2}}\right] z^{2} } \\
& -2 k \frac{\rho g}{\mu \omega}\left(\frac{\mathrm{d} \mu}{\mathrm{d} \tau}+1\right) z^{3}+(1+\tau / \mu) z^{4}=0 .
\end{aligned}
$$

Introducing (27) into the interface conditions (38), with $z_{1}, z_{2}$ extracted from (39), one obtains

$$
\begin{aligned}
& \llbracket A_{1}+A_{2} \rrbracket=0 \\
& \llbracket z_{1} A_{1}+z_{2} A_{2} \rrbracket=0 \\
& \llbracket \mu(1+\tau / \mu)\left(z_{1}^{2} A_{1}+z_{2}^{2} A_{2}+A_{1}+A_{2}\right) \rrbracket=0 \\
& \llbracket\left[\mu \left[\left(4 \mu / \mu-1+\tau / \mu-\frac{\rho \xi^{2}}{\mu \omega^{2}}\right)\left(z_{1} A_{1}+z_{2} A_{2}\right)-(1+\tau / \mu)\left(z_{1}^{3} A_{1}+z_{2}^{3} A_{2}\right)\right.\right. \\
& \left.\quad+k \frac{\rho g}{\mu \omega}\left(\frac{\mathrm{d} \mu}{\mathrm{d} \tau}+1\right)\left(z_{1}^{2} A_{1}+z_{2}^{2} A_{2}+A_{1}+A_{2}\right)-\frac{\rho g}{\mu \omega}\left(A_{1}+A_{2}\right)\right] \rrbracket=0 .
\end{aligned}
$$

These conditions may once again be cast in the form (32) upon making use of (30) and (31), the coefficients $C_{11}, C_{12}, C_{21}$ remaining the same, but $C_{22}$ becoming

$$
\begin{aligned}
C_{22}=\left[\mu \left[\left(4 \mu / \mu-1+\tau / \mu-\frac{\rho \xi^{2}}{\mu \omega^{2}}\right)-(1+\tau / \mu)\left(z_{1}^{2}+z_{1} z_{2}+z_{2}^{2}\right)\right.\right. \\
\left.\left.+k \frac{\rho g}{\mu \omega}\left(\frac{\mathrm{d} \mu}{\mathrm{d} \tau}+1\right)\left(z_{1}+z_{2}\right)\right]\right] .
\end{aligned}
$$

Equation (33) may now be solved numerically for the minimum value $\xi_{m}^{2}$ with coefficients $C_{i j}$ and roots $z_{\gamma}$ being determined by (32) and (41), respectively. For given material properties and a given state of stress it is thus possible to say whether or not an interfacial perturbation with wavenumber $\omega$ will be unstable $\left(\xi^{2}<0\right)$. The bifurcation wavenumber $\omega_{c}$ corresponds to $\xi_{m}^{2}=0$, as is evident from the above relations. Therefore, a graph of the relationship between the critical wavenumber (or wavelength) and the prestress at the interface will separate regions of stability and instability in the wavenumber-prestress plane, as will be seen in the following.

\section{Spfotiat Casfos}

Although the bifurcation and stability criteria that have been derived allow the critical wavenumber $\omega_{c}$ and the minimum eigenvalue $\xi_{m}^{2}$ to be determined from the 
solution of a highly complicated implicit equation, it is possible to give explicit analytic results for certain special cases of interest, two of which will now be discussed. In each case an elastoplastic half-space is considered to rest on a fluid. The stiffness of the lower half-space is thus ignored, i.e. $\mu^{b}={ }_{\mu}^{*}=0$ and one can put $\mu=\mu^{a}, \stackrel{*}{\mu}=\stackrel{*}{\mu}^{a}$.

The case $k=0$. This corresponds to the situation in which the principal stress difference in each half-space remains constant and independent of $x_{2}$. Equation (28) then becomes a biquadratic equation in $z$, with roots

$$
\begin{aligned}
& z_{1,2}=\left\{\frac{2 \mu / \mu-1}{1+\tau / \mu} \pm\left[\left(\frac{2 \mu / \mu-1}{1+\tau / \mu}\right)^{2}-\frac{1-\tau / \mu}{1+\tau / \mu}\right]^{1 / 2}\right\}^{1 / 2}, \quad \text { if } 4 *(\mu-\stackrel{*}{*})<\tau^{2}, \\
& z_{1,2}=\left\{\frac{1}{2}\left[\left(\frac{1-\tau / \mu}{1+\tau / \mu}\right)^{1 / 2}+\frac{2 * / \mu-1}{1+\tau / \mu}\right]\right\}^{1 / 2} \pm i\left\{\frac{1}{2}\left[\left(\frac{1-\tau / \mu}{1+\tau / \mu}\right)^{1 / 2}-\frac{2 * / \mu-1}{1+\tau / \mu}\right]\right\}^{1 / 2}, \\
& \text { if } 4 \stackrel{*}{\mu}(\mu-\stackrel{*}{\mu}) \geqslant \tau^{2} \text {. }
\end{aligned}
$$

The above results apply to the half-space $a$, where $\operatorname{Re}\left(z_{;}\right)>0$ according to (27). If the lower half-space is of interest and is modelled as a solid, one simply reverses the signs of the roots in (42).

The roots (42) do in fact allow an explicit determination of the critical wavenumber $\omega_{c}$. Thus, after computation of the matrix coefficients $C_{11}, C_{12}, C_{21}, C_{22}$ in (32), the bifurcation criterion (33) yields

$$
\frac{\Delta \rho g}{2 \mu \omega_{c}}=\frac{1}{S}\left[\left(\frac{1-\tau / \mu}{1+\tau / \mu}\right)^{1 / 2}(2 \mu / \mu+\tau / \mu)-\tau / \mu\right], \quad S \equiv z_{1}+z_{2},
$$

where the $z_{1,2}$ are given by (42) and $\Delta \rho \equiv \rho^{a}-\rho^{h}$. Here $\mu$ as well as $\stackrel{*}{\mu}$ are of course taken to be functions of $\tau$. Also, the superscript $a$ has been deleted, it being understood that - with the exception of $\Delta \rho g / \omega_{c}$ - in (43) and subsequent expressions all indexfree variables and functions are defined on the side of the interface belonging to halfspace $a$.

Since for the bifurcation of interest the half-space $a$ has to be in the elliptic regime, as explained in the discussion following (28), the denominator $S$ in (43) is always strictly positive; moreover, for $\tau=0$ the term within brackets becomes constant and equal to 2. These observations imply that for a denser upper half-space $(\Delta \rho>0)$ a bifurcation is always possible, while for a denser lower half-space $(\Delta \rho<0)$ a hydrostatically stressed overburden cannot bifurcate. For large enough values of $|\tau|$ the rightl-hand side of (43) can vanish. The vanishing of the numcrator in (43) yiclds BIOT's (1965) condition for the surface bifurcation of an (orthotropic) half-space that is subjected to a lateral stress $\left(-\sigma_{1}\right)$ and it confirms Biot's prediction that the corresponding critical wavelength is zero.

For $\tau=0, \stackrel{*}{\mu}(0)=\mu(0)=G$, where $G$ is the linear elastic shear modulus, (43) yields the interesting result

$$
\frac{\Delta \rho g}{2 \mu(0) \omega_{c}}=1
$$


This shows that when a hydrostatically stressed elastic half-space overlies a less dense, incompressible fluid, the critical wavelength for a bifurcation is likely to be very large. Thus, if the quantity $\left(-\sigma_{2}\right) / \rho g$ is taken as a measure of overburden thickness in a geological setting, then the ratio critical wavelength/overburden thickness will be of the order of $2 \mu /\left(-\sigma_{2}\right)$ or between $10^{2}$ and $10^{4}$, when $\mu$ is an elastic shear modulus.

As was established in the general analysis of the previous section, the graph of the critical wavelength (43) contains all neutral equilibrium points $\left(\xi_{m}^{2}=0\right)$ in the $(\Delta \rho g / 2 \mu \omega)-(\tau / \mu)$ space. This "critical-wavelength curve" therefore separates a region of stable perturbation wavelengths from a region of unstable perturbation wavelengths, which in the following will be referred to as "stable" and "unstable" regions, respectively.

When $k=0$, a simple way of discriminating between the stable and unstable side of the critical-wavelength curve is by calculating $\rho \xi^{2} / \mu \omega^{2}$ for points on the $\Delta \rho g / 2 \mu \omega$ axis in the neighbourhood of the point $(\tau / \mu=0, \Delta \rho g / 2 \mu(0) \omega=1)$. Since $\mu(0) / \mu(0)=1$ by assumption, the roots of interest of (39) are

$$
z_{1}=1, \quad z_{2}=(1-\beta)^{1 / 2}, \quad \beta \equiv \rho \xi^{2} / \mu \omega^{2} .
$$

Using these in (32) and (33) and recalling that $C_{22}$ must be determined from (41) for the stability analysis, one arrives at the following equation for $\beta$ at points with $\tau / \mu=0$, $\Delta \rho g / 2 \mu(0) \omega=1+\varepsilon$, where $|\varepsilon| \ll 1$ :

$$
\left(1-\frac{\Delta \rho}{2 \rho}\right) \beta+1+\varepsilon-(1-\beta)^{1 / 2}\left(1-\varepsilon-\frac{\Delta \rho}{2 \rho} \beta\right)=0 .
$$

An asymptotic solution for $\beta$ is of the form

$$
\beta=-\frac{4}{3} \varepsilon+\mathrm{O}\left(\varepsilon^{2}\right),
$$

indicating that for $\varepsilon>0(\Delta \rho g / 2 \mu(0) \omega>1)$ the interface is unstable. This result does not come as a surprise since the gravitational potential energy released by perturbation wavelength beyond the critical exceeds the concomitant elastic energy stored in the solid layer. Along with this determination of $\beta$ one must of course ensure that all other eigenvalues - found by solving (32), (39), (41) numerically - are in fact larger.

The case $|k \rho g / \mu \omega| \ll 1$. This short-wavelength limit has the following significance. From (18), (19) and the constitutive relation (14) it is easily seen that the gradient of the normal strain $\varepsilon_{11}$ in the (vertical) $x_{2}$ direction is given by $\mathrm{d} \varepsilon_{1 /} / \mathrm{d} x_{2}=-k \rho g / 2 \mu(\tau)$. The condition may therefore be expressed as $\left|2 \mu\left(\mathrm{d} \varepsilon_{1 /} / \mathrm{d} x_{2}\right) / \mu \omega\right| \ll 1$. Moreover, since $2^{*} \mu / \mu=\mathrm{O}(1)$ for most applications, this is equivalent to $\left|\left(\mathrm{d} \varepsilon_{1 /} / \mathrm{d} x_{2}\right) L\right| \ll 1$, where $L=2 \pi / \omega$ is the wavelength of the mode in the $x_{1}$ direction. Hence, with

$$
\lambda \equiv k \frac{\rho g}{\mu \omega},
$$

the asymptotic analysis for $|\lambda| \ll 1$ applies when strain gradients in the $x_{2}$ direction are much smaller than the inverse of the eigenmode's wavelength in the $x_{1}$ direction.

The roots $z_{1}, z_{2}$ of (28) that are required for evaluating (32) and the bifurcation condition (33), are found, in the case $|\lambda| \ll 1$, with the aid of a straightforward 
asymptotic expansion of (28) with respect to $\lambda$

$$
z_{\gamma}(\hat{\lambda})=z_{\gamma}+\frac{(\mathrm{d} \mu / \mathrm{d} \tau+1) z_{i}^{2}-\left(2 \mathrm{~d}^{*} \mu / \mathrm{d} \tau-\mathrm{d} \mu / \mathrm{d} \tau\right)}{2\left[(1+\tau / \mu) z_{\gamma}^{2}-(2 \mu / \mu-1)\right]} \dot{\lambda}+\mathrm{O}\left(\lambda^{2}\right),
$$

where the $z_{\gamma}$ are given by (42). Upon entering these into (32) and cvaluating (33) by collecting terms of like order in $\lambda$, one obtains, after lengthy but straightforward algebraic manipulations and use of (42), the following expression for the critical wavenumber $\omega_{c}$, correct to the first order in $\lambda$ :

$$
\frac{\Delta \rho g}{2 \mu\left(\omega_{c}\right.}=\frac{[(1-\tau / \mu) /(1+\tau / \mu)]^{1 / 2}(2 \mu / \mu+\tau / \mu)-\tau / \mu}{S\left\{1-\left[k /\left(1-\rho^{h} / \rho^{a}\right)\right]\left[2 \mathrm{~d}^{*} / \mathrm{d} \tau-\mathrm{d} \mu / \mathrm{d} \tau+(\mathrm{d} \mu / \mathrm{d} \tau+1)\left(2+[(1-\tau / \mu) /(1+\tau / \mu)]^{1 / 2}\right)\right\}^{\prime}\right.}
$$

where $S$ is defined as in (43). It should be kept in mind, however, that for large prebifurcation strains $\tau / \mu$ cannot be negiected and the correction terms, $\mathrm{d} \mu / \mathrm{d} \tau$ and $\mathrm{d} \mu / \mathrm{d} \tau$ can also become important.

\section{Discussion of Results for Two Particular Materials}

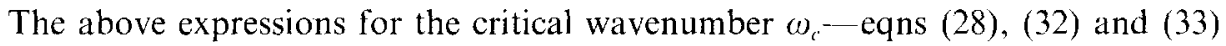
for the general case as well as (43) and (50) for the two special cases-are valid for any incompressible, pressure insensitive, orthotropic material that obeys a constitutive relation of the general form of equation (14). It is nevertheless clear that numerical values for the critical wavenumber will strongly depend on the particular form of the functions $\mu(\tau)$ and $\mu(\tau)$ in (18). Of the many different possibilities, two relatively simple models will be explored.

The first material to be studied is a Mooney-Rivlin hyperelastic material, which, under plane strain conditions satisfies the stress--strain relation

$$
\tau / G=\sinh \left(\varepsilon_{22}-\varepsilon_{11}\right)
$$

Here $G$ is the material's initial shear modulus and $\varepsilon_{11}, \varepsilon_{22}$ are the logarithmic strains in the $x_{1}$ and $x_{2}$ directions, respectively. The pure shear stress-strain curve for the Mooney-Rivlin material is depicted in Fig. 2.

The plane strain incremental moduli $\mu(\tau)$ and $\stackrel{*}{\mu}(\tau)$ defined by (14) are given by

$$
\mu(\tau)=\stackrel{*}{\mu}(\tau)=G\left[1+(\tau / G)^{2}\right]^{1 / 2}
$$

for this material.

The second material to be considered is a hypoelastic Stören-Rice material [see STÖREN and RICE (1975)], the stress--strain relation of which is given by the discontinuous power law 


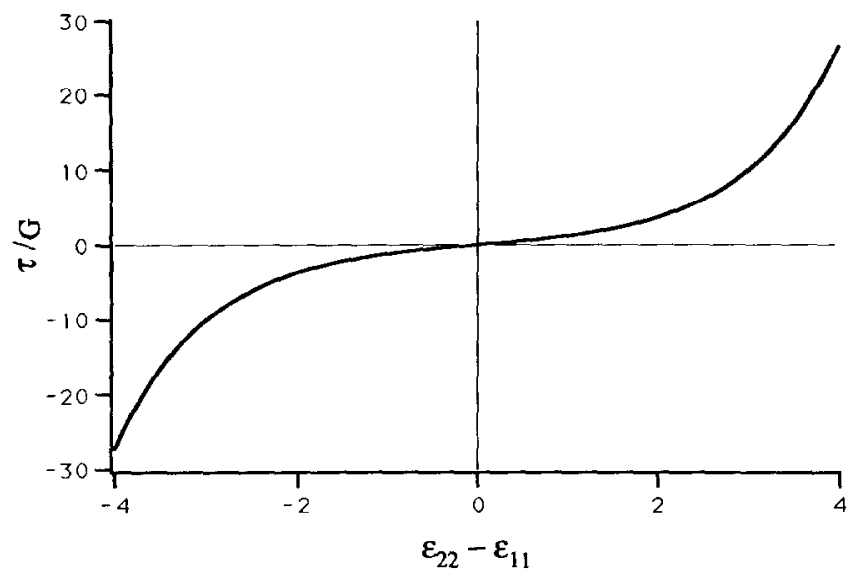

FIG. 2. Stress-strain relation for Mooney-Rivlin material.

$$
\tau / \tau_{y}=\left\{\begin{array}{lll}
G\left(\varepsilon_{22}-\varepsilon_{11}\right) / \tau_{y}, & \text { if } & |\tau| \leqslant \tau_{y}, \\
{\left[G\left(\varepsilon_{22}-\varepsilon_{11}\right) / \tau_{y}\right]^{1 / m},} & \text { if } & |\tau|>\tau_{y} .
\end{array}\right.
$$

Here $m$ denotes a hardening modulus ( $m>1$ ), while $\tau_{y}$, is the yield stress in pure shear. This type of stress-strain relation is shown in Fig. 3.

The corresponding expressions for the incremental moduli are given by

$$
\begin{array}{ll}
\mu(\tau)=\stackrel{*}{\mu}(\tau)=G, & \text { if }|\tau| \leqslant \tau_{y}, \\
\mu(\tau)=G\left|\tau / \tau_{y}\right|^{1-m}, \quad \stackrel{*}{\mu}(\tau)=\mu(\tau) / m, & \text { if } \quad|\tau|>\tau_{y} .
\end{array}
$$

The fundamental difference in the behaviour of the two materials is that the functions

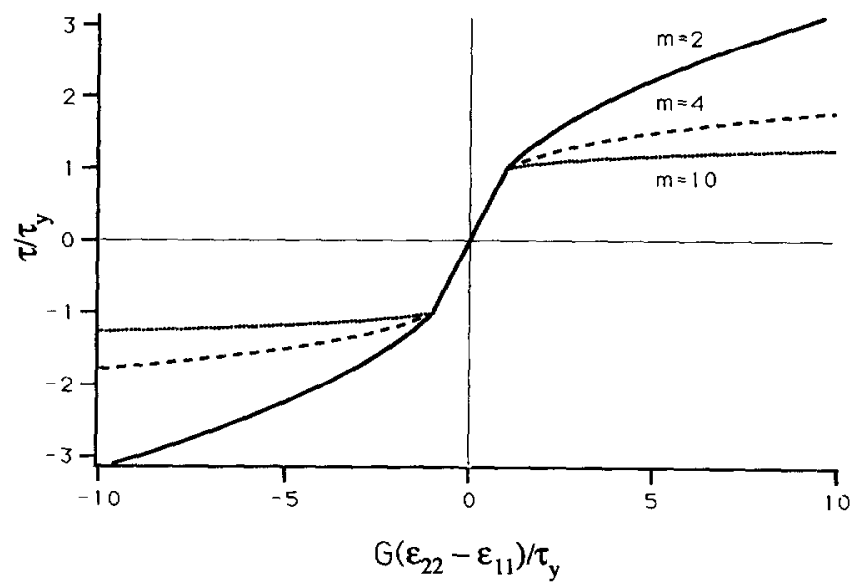

Frg. 3. Stress strain relation for Stören-Rice material. 
$\mu(\tau)$ and $\stackrel{*}{\mu}(\tau)$ increase with $\tau$ for the Mooney-Rivlin material (52), but decrease with $\tau$ for the Stören-Rice power law material (54).

Taking either (52) or (54) as material law, the critical wavenumber $\omega_{i}$ may now be calculated from (33) or-in the limit $|\hat{\lambda}| \ll 1$-from (50) upon substitution for $\mu(\tau)$ and $* \dot{\mu}(\tau)$ from either (52) or (54). For a given gradient $\mathrm{d} \tau / \mathrm{d} x_{2}=k \rho g$ of the stress deviator and a given density ratio $\rho^{b} / \rho^{u}$, eqn (33) is solved numerically with the aid of a straightforward bisection method. The exact results obtained in this manner are presented together with the asymptotic results in Fig. 4, for the Mooney-Rivlin material, and Figs 5 and 6 for a Stören-Rice material with the hardening exponents $m=2$ and $m=4$, respectively. In all figures a dimensionless critical wavelength has been plotted against a dimensionless stress deviator for various values of the parameter $k$, assuming the fixed density ratio $\rho^{b} / \rho^{a}=0.8$. Thus, $k=0,-0.03,0.03$ in Figs 4 a$c$, while $k=0,-0.015,0.015$ in Figs $5 \mathrm{a}-\mathrm{c}(m=2)$ and Figs $6 \mathrm{a}-\mathrm{c}(m=4)$. For the Mooney-Rivlin material the dimensionless wavelength and stress deviator are defined as $\Delta \rho g / 2 G \omega_{c}$ and $\tau / G$, respectively, while for the Stören-Rice material the corresponding quantities are defined as $\Delta \rho g / 2 \tau_{y} \omega_{c}$ and $\tau / \tau_{y}$, respectively. In all calculations performed for the Stören-Rice material the value $\tau_{y} / G=10^{-3}$ was assumed for the yield strain in simple shear.

Some general remarks on the results shown in Figs 4-6 are now in order. First, the determination of the character of the equilibrium states adjacent to the criticalwavelength curves, i.e. the delineation of regions of stability and instability, is based on the sign criterion for the minimum eigenvalue $\xi_{m}^{2}$ of the linearized perturbation problem; these results were computed numerically for a number of interior points in each region. Thus, for a given state of stress in the unperturbed half-spaces, perturbations with wavelengths that lie on one side of the critical curve are stable, that is will not grow with time, but decay in the presence of the slightest amount of viscous dissipation, while perturbations with wavelengths on the other side of the critical curve are unstable and will tend to grow, although there remains the possibility of the perturbed system evolving towards a new, postbifurcation equilibrium state that will be characterized by a finite-amplitude disturbance of the layer interface. The existence of such equilibrium states in the postbifurcation regime may be expected for solid/fluid two-layer systems from the fact that the solid possesses an elastic range and finite strength. Such states are of considerable interest, for example in geological or geotechnical studies of saltdoming instabilities, but the conditions for their existence as well as their exact nature have heen studied only little.

Secondly, we recall that negative values of $\Delta \rho g / 2 G \omega_{c}$ or $\Delta \rho g / 2 \tau_{v} \omega_{c}$ correspond to a gravitationally stable density stratification $\left(\Delta \rho \equiv \rho^{a}-\rho^{h}<0\right)$. For this case and under the additional restriction of a vanishing stress gradient $\mathrm{d} \tau / \mathrm{d} x_{2}=0(k=0)$ we shall recover certain earlier results by BIOT $(1963 \mathrm{~b}, 1965)$.

Finally, it will be clear that an unstably stratified two-layer system of infinite lateral extent can indeed be destabilized by any prevailing stress state. Nevertheless, the results of this study can be meaningfully applied to overburden/substratum interfaces of finite extent that delimit the range of critical wavelengths by their width. Thus, for finite systems, the quantities of interest will be the critical load required for activating the maximum available wavelength or, alternatively, the critical wavelength associated with a given state of stress. 
Interfacial instability
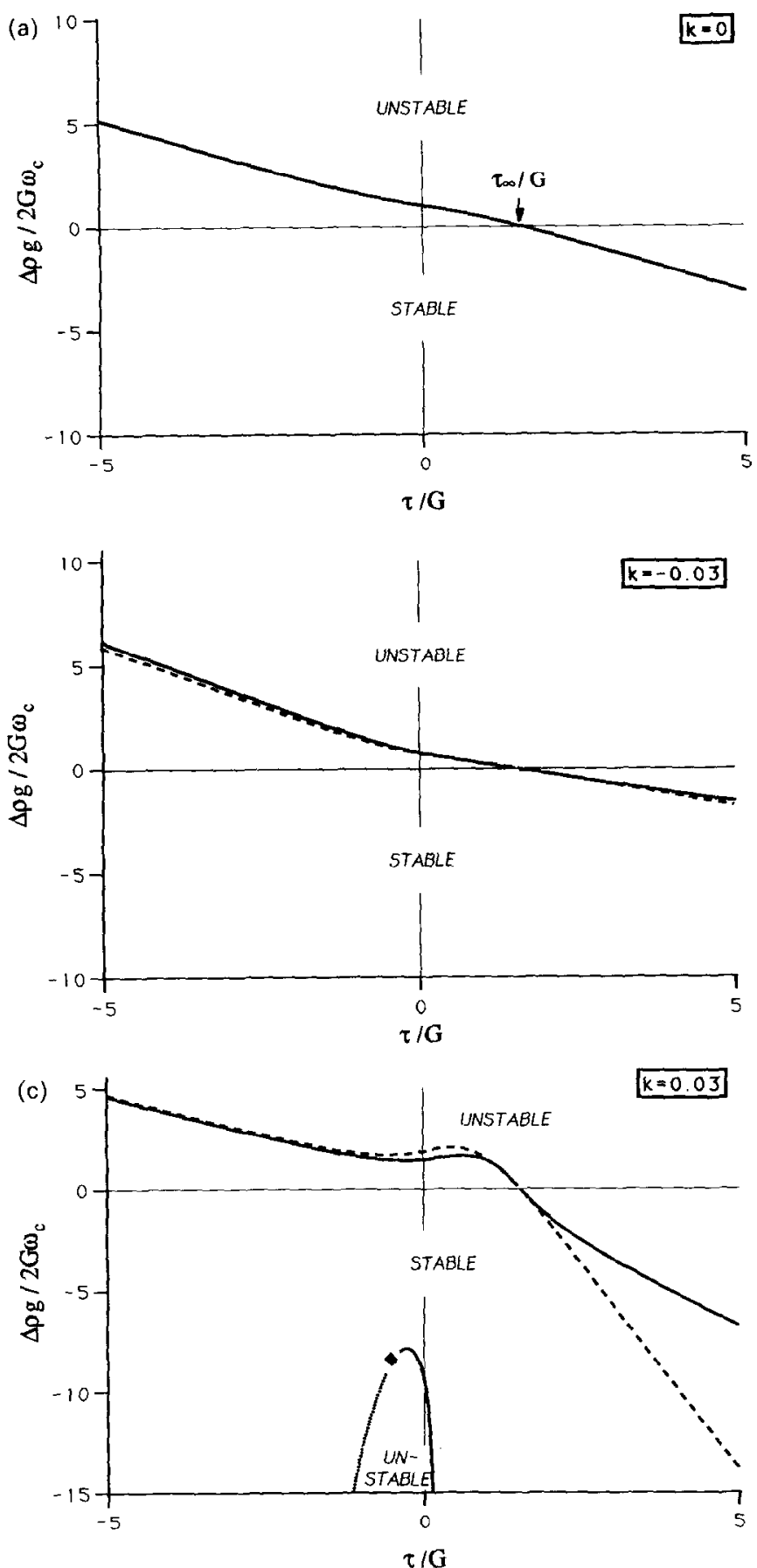

Fig. 4(a)-(c). Critical wavelength versus initial stress at interface between Mooney-Rivlin material and inviscid fluid, showing effect of different stress gradients $k$; dashed line is asymptotic solution $(50)$; marks limit of decaying solutions. 

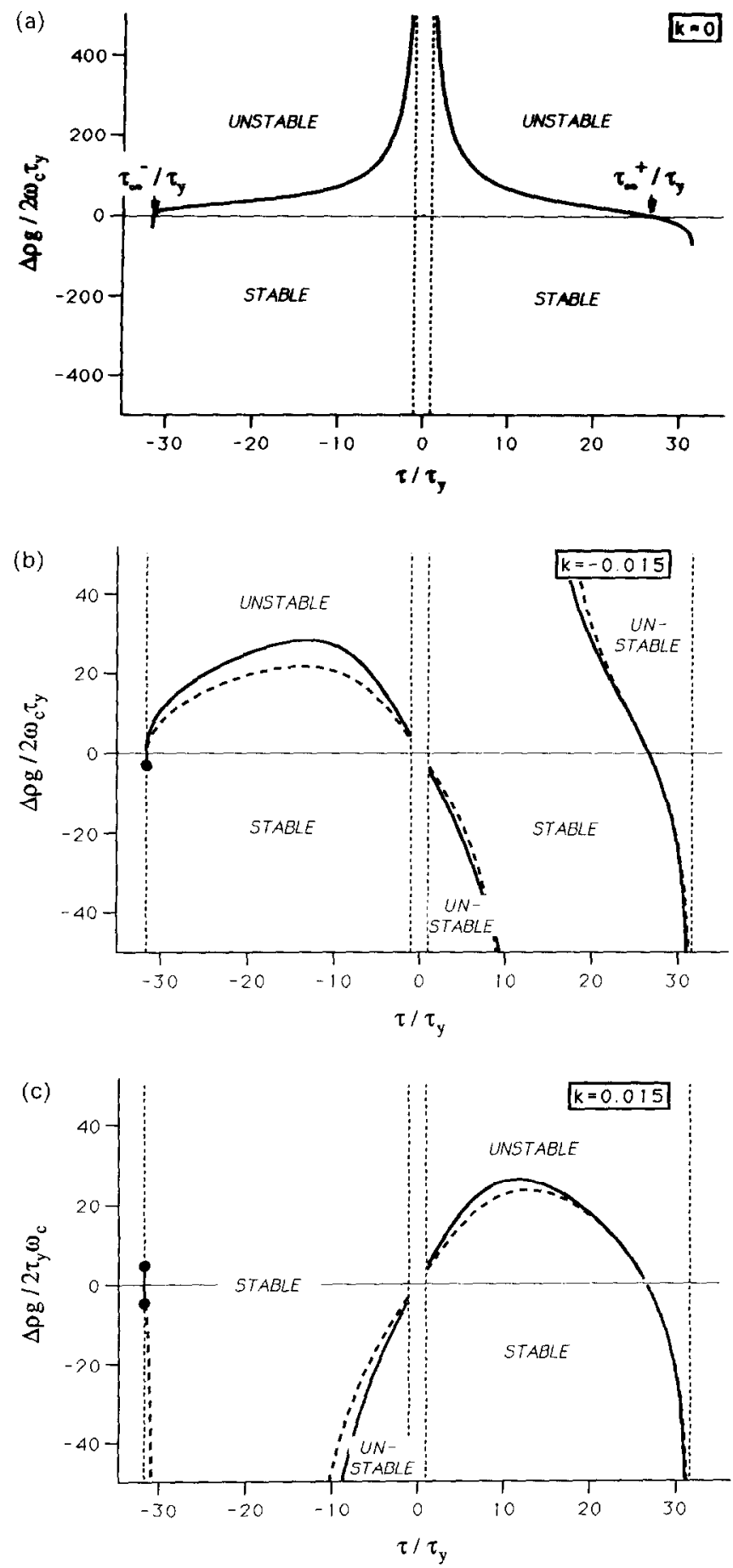

FIG. 5(a)-(c). Critical wavelength versus initial stress at interface between Stören-Rice material $(m=2)$ and inviscid fluid, showing effect of different stress gradients $k$; dashed line is asymptotic solution (50):

- marks loss of ellipticity of eqn (23). 

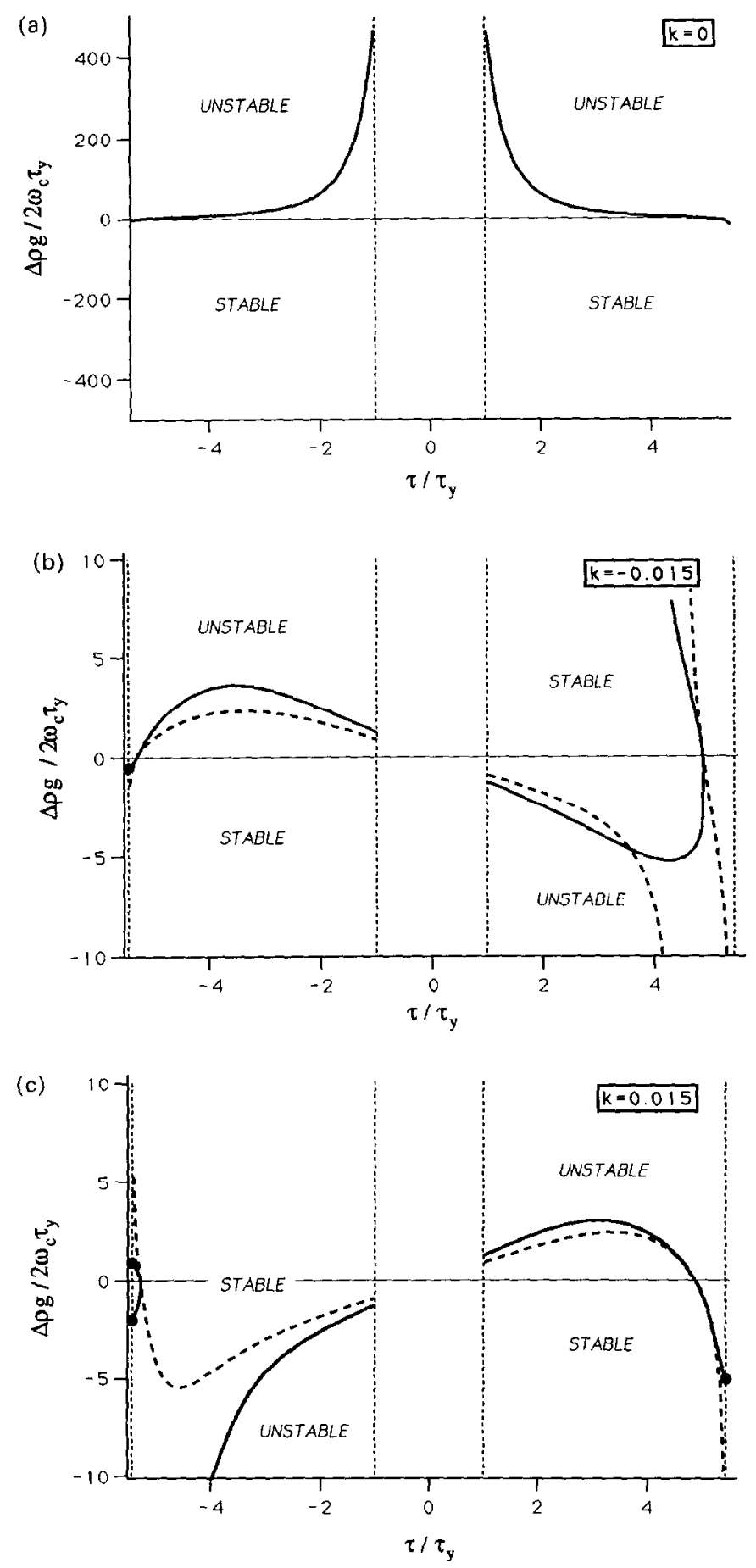

FIG. 6(a)-(c). Critical wavelength versus initial stress at interface between Stören-Rice material $(m=4)$ and inviscid fluid, showing effect of different stress gradients $k$; dashed line is asymptotic solution (50); - marks loss of ellipticity of eqn (23). 
Consider now the behaviour of a half-space composed of a Mooney-Rivlin material. Figure $4 a$ gives the dimensionless critical wavelength as a function of the dimensionless stress deviator at the interface when the latter remains uniform throughout the half-space $(k=0)$. The stable states lie below and the unstable states above the curve. The zero-wavelength intercept on the stress axis determines the stress level required for inducing a pure surface bifurcation mode. This surface bifurcation was discussed by BIOT $(1963 \mathrm{~b}, 1965)$ in his study of a gravitationally stable halfspace; his results for $k=0$ and $\Delta \rho \leqslant 0$ are reproduced by the corresponding segment of the critical curve in Fig. 4a. For $\Delta \rho>0$, the zero-stress intercept of the critical curve determines the critical wavelength that will be destabilized by gravity alone, all shorter wavelengths requiring some compression for their appearance.

The stress deviator $\tau / G$ affects the critical wavelength in a twofold manner. First, if $\tau$ is positive (negative), through the progressive destabilization (stabilization) of the system with increasing (decreasing) $\tau / G$ or increased (decreased) lateral compression of the layer. This is a purely geometric effect that is responsible for the instability of solids under compression, with examples ranging from Euler columns and plate buckling in structures to surface instabilities on half-spaces arising from surface-parallel compression in finitely strained solids (BIOT, 1963b, 1965). The second effect is the progressive stiffening of the material, i.c. the increase of its incremental moduli, with increasing $|\tau| / G$. The two effects cooperate when $\tau$ is negative, but counteract each other for positive $\tau$ when indeed the destabilizing influence of lateral compression becomes dominant at $\tau>\tau_{\infty}$, where $\tau_{\infty}$ is Biot's critical stress for the appearance of a surface instability on a weightless half-space. For the Mooney-Rivlin material, the asymmetric geometric effect of stabilizing lateral tension and destabilizing lateral compression dominates the symmetric constitutive stiffening effect; the resulting graph of critical wavelength versus stress deviator is therefore skew symmetric with respect to the zero stress axis. For the further interpretation of Fig. 4 it is therefore best to think about the critical curve in terms of the critical compressive stress required to destabilize a given wavelength, if $\tau>0$ and in terms of the critical tensile stress required to stabilize a given wavelength, if $\tau<0$.

The effect of a negative stress deviator gradient $(k=-0.03)$ on the stability of a half-space of Mooney-Rivlin material is apparent from Fig. $4 b$. Three regions of the critical wavelength versus stress deviator graph can be distinguished, according to the prevailing stabilization or destabilization mechanism. For $\Delta \rho>0$ and $\tau<\tau^{*}<0$, where $\tau^{*}$ is defined by $\omega_{c}\left(\tau^{*} ; k=-0.03\right)=\omega_{c}\left(\tau^{*} ; 0\right)$, the material stiffness increases away from the interface due to the increase in $|\tau|$. The increase in the interface-parallel tensile stress provides a geometric stabilization. Due to these stabilization mechanisms, the critical wavelength for a given value of $\tau$ is higher than its counterpart for $k=0$. The interfacial stability is thus increased in this region by a negative stress gradient $k$. For $\Delta \rho>0$ and $\tau=0$, the decrease in $\tau$ (or increase in lateral tension) away from the interface stiffens the material and reduces the critical mode's decay length. This in turn reduces the critical wavelengths in the region $\tau^{*}<\tau<\tau_{\infty}$ around $\tau=0$ relative to the wavelengths for $k=0$. If $\Delta \rho<0$ and when $\tau$ exceeds $\tau_{\infty}$, it decreases away from the interface and the associated geometric stabilization requires more compression for destabilizing a given wavelength than is needed for $k=0$. Again, therefore, the interface is stabilized by a negative $k$. It will further be noted that there is good agreement 
between the exact numerical solution of (32) and (33) and the asymptotic results derived from (50), due to the fact that the value of $|\lambda|=\left|k \rho g / \mu(\tau) \omega_{c}\right|$ remains sufficiently small over a wide range of $\tau$-values.

Figure $4 c$ illustrates the effect of a positive stress deviator gradient $(k=0.03)$. Three regions may again be distinguished. For $\Delta \rho>0$ and $\tau<\tau^{*}<0$, where $\omega_{c}\left(\tau^{*}, k=0.03\right)=\omega_{c}\left(\tau^{*}, 0\right)$, the material becomes softer with increasing distance from the interface, since $|\tau|$ decreases. The lateral tension required for stabilizing a given wavelength is higher than that for $k=0$. Equivalently, the critical wavelength for a given value of $\tau$ is lower than the corresponding wavelength for $k=0$. The interfacial stability is thus decreased in this region by a positive stress gradient $k$. In the small region $\tau^{*}<\tau<\tau_{\infty}$ around the point $\tau=0$, the increase of $\tau$ away from the interface increases the critical mode's decay length and has a stabilizing effect on the system leading to higher critical wavelengths. Eventually the destabilizing effect of the increasing lateral compressive stress dominates and the critical wavelength vanishes at $\tau_{\infty}$. For $\Delta \rho<0$ and $\tau>\tau_{\infty}, \tau$ increases away from the interface and the associated geometric effect of increased axial compression of the half-space permits a smaller positive $\tau$ to destabilize a given wavelength, than would be required for $k=0$. The asymptotic results obtained for this case from (50) are in good agreement with the results obtained from (32) and (33) near $\tau_{\infty}$, where the critical wavelength is small, and at large absolute value of $\tau$ where the incremental moduli acquire large values, resulting in small values of $|\lambda|=\left|k \rho g / \mu(\tau) \omega_{c}\right|$ in these cases.

Apart from the solution branch that passes through $\tau_{\infty}$ and is expected from the results for $k=0,-0.03$, a second branch appears near $\tau=0$, if the density stratification of the system is stable $(\Delta \rho<0)$. The reason for this instability is the following. The increase in lateral compression away from the interface destabilizes the system and there exists a mode with an adequately large decay length that releases more energy than the potential energy required to lift the stably stratified interface. Any wavelength that is larger than this critical wavelength will thus be unstable, as is verified by a linearized stability analysis. This second branch of the critical curve has a vertical asymptote at some positive value of $\tau$ close to $\tau=0$, which reflects the stabilizing influence of material stiffening; the branch ends at some value $\tau<0$ that marks the emergence of solutions with infinite decay length.

Figures 5 and 6 give the dimensionless critical wavelength $\Delta \rho g / 2 \tau_{y} \omega_{c}$ as a function of the dimensionless stress deviator $\tau / \tau_{y}$ for the case of two variations of the StörenRice hypoelastic material, one with a higher hardening ( $m=2$ in Fig. 5) and the other with lower hardening ( $m=4$ in Fig. 6 ) in their uniaxial stress--strain response. In all the graphs presented here the results are given outside the elastic zone $\left|\tau / \tau_{y}\right| \leqslant 1$. Due to the discontinuity of the incremental modulus $\mu(\tau)$ at $\left|\tau / \tau_{y}\right|=1$ and the subsequent rapid decline of both the incremental moduli $\mu(\tau)$ and $\mu(\tau)$ [see (54)] for $\left|\tau / \tau_{y}\right|>1$ there is a difference of the order of $G / \tau_{y}$ between the critical wavelength values inside and outside the elastic zone. Indeed, for $\left|\tau / \tau_{y}\right| \leqslant 1$ the wavelengths agree essentially with the corresponding wavelengths for the Mooney-Rivlin material in the neighbourhood of $\tau=0$, and for stresses of the order of $\tau_{y} / G$ the effect of the difference in constitutive response is negligible. The calculations are terminated at values of $\tau / \tau$, such that the governing equations become hyperbolic [cf. the discussion of eqns (23) and (28)]. This loss of ellipticity represents an additional feature of a Stören-Rice 
material which not only is destabilized in both tension and compression, but also admits shear band modes of localized deformation at appropriate levels of stress, as was discussed by STÖREN and RICE (1975). By contrast, it can easily be seen that for a Mooney-Rivlin material (23) will remain elliptic at all stress levels.

In Fig. 5a a plot of the critical wavelength versus the stress deviator is shown for a Stören-Rice material with hardening exponent $m=2$ for the case of a vanishing gradient of $\tau$ in the $x_{2}$ direction $(k=0)$. As discussed previously, an increase in $\tau$ will destabilize the layer, while a decrease will have the opposite effect. This asymmetric effect is independent of the constitutive law and controlled by the sign of $\tau$. The constitutive response of the layer is symmetric in $\tau$, since it depends upon $|\tau|$ only and the rapid decrease in stiffness of a Stören-Rice material with increasing $|\tau|$ has a destabilizing effect that increases with the hardening exponent $m$. The behaviour of the critical wavelength as a function of the stress deviator as shown in Fig. 5a is clearly dominated by the symmetric constitutive response. As a consequence, a zerowavelength surface instability appears both under compressive as well as tensile lateral stress at $\tau_{\infty}^{+}>0$ and $\tau_{\infty}<0$, respectively. The asymmetric effect of $\tau$ or $\sigma_{1}$ (assuming $\sigma_{2}$ remains fixed) expresses itself in the steeper decline of the positive branch of the critical curve, resulting in $\left|\tau_{\infty}^{+}\right|<\left|\tau_{\infty}^{-}\right|$for the surface instability. The graph of the dimensionless critical wavelength remains continuous across the clastic rangc $-1<\tau / \tau_{y}<1$, where $\Delta \rho g / 2 \tau_{y} \omega_{c} \approx G / \tau_{y}=10^{3}$.

The effect of a negative gradient in $\tau$ on the stability of the half-space is shown in Fig. 5b. The graph of the critical wavelength has lost its symmetry, despite the persistence of zero-wavelength surface instabilities in tension and compression at the previous critical loads $\tau_{x}^{-} / \tau_{y}$ and $\tau_{x}^{+} / \tau_{y}$, respectively. Thus, in the case of gravitationally unstable density stratification $(\Delta \rho>0)$ there exists a finite critical wavelength for all $\tau_{\infty}^{-}<\tau \leqslant-\tau_{y}$, but only within a certain interval $\tau_{y} \leqslant \tau<\tau_{x}^{+}$. For $\Delta \rho<0$ the $\tau \geqslant \tau_{\text {s }}$ branch reaches some maximum value of $\tau$ outside the range of the figure, the complex behaviour of the critical curve in this quadrant depending sensitively upon the value of $m$ as a comparison with Fig. 6b will show. It is of interest to note that the two branches of the asymptotic solution shown in Fig. $5 b$ for positive $\tau$ are separated by a vertical asymptote (not shown here). The behaviour of the critical curve near the origin may be understood in terms of a hypothetical material defined by a smooth continuation of the power-law relation in (54) to zero stress, where such a material will become infinitely stiff. As the asymptotic result (50) shows, the conditions $\mathrm{d} \mu / \mathrm{d} \tau$, $\mathrm{d} \mu / \mathrm{d} \tau \rightarrow \infty$ as $\tau \rightarrow 0$ implying a vanishing critical wavelength. Associated with this hypothetical material will be a smooth continuation of the critical curve across the yield stress $\pm \tau_{y}$ and through the coordinate origin of Figs $5 \mathrm{~b}, \mathrm{c}$ and $6 \mathrm{~b}, \mathrm{c}$. This shows that the sharp discontinuity exhibited by the actual critical curve at the yield stress originates from the discontinuity in the tangent modulus of the Stören-Rice material.

The behaviour of the critical curve in Fig. $5 \mathrm{~b}$ can be explained in terms of the combined effects of the interface-parallel stress (for a fixed normal stress) and the rate of decrease $k$ of the normal stress difference $\tau$ away from the interface. When $\tau$ is negative and small in absolute value, the latter will increase away from the interface and add a further stabilizing influence, due to the stabilizing effect of a tensile lateral stress; this leads to critical wavelengths that increase with $|\tau|$ within some range of negative $\tau$ values. However, beyond a certain absolute value of $\tau$ the destabilizing 
stiffness reduction associated with high values of $|\tau|$ again dominates the stabilizing effects of interface-parallel tension and increasing stiffness away from the interface, leading eventually to a surface bifurcation instability mode in tension at $\tau_{\infty}$, which is unaffected by $k$ and coincides with the critical tensile stress for $k=0$ of Fig. 5a. At positive values of $\tau$ the destabilizing effect of interface-parallel compression is counteracted by the decrease in $|\tau|$ away from the interface $(k<0)$. As a consequence, for $\tau \geqslant \tau_{y}$, the critical wavelength for a gravitationally unstable stratification $\Delta \rho>0$ is much larger than its counterpart value for $-\tau$ (outside the scale of the graph), except for values of $\tau$ close enough to the critical load $\tau_{\infty}^{+}$where the influence of $k$ ceases to be felt. Notice also that for $\tau \geqslant \tau_{y}$ any sufficiently large wavelength perturbation of a gravitationally stable interface $(\Delta \rho<0)$ will be unstable as a consequence of increasing layer-parallel compression away from the interface.

Figure $5 \mathrm{c}$ illustrates the effects on interfacial stability of a decrease in the stress deviator away from the interface. As expected, the graph represents roughly a mirror image of Fig. 5b.

Analogous results for the more rapidly softening Stören-Rice material $(m=4)$ are shown in Fig. 6.

\section{Conclusions}

The instability of a solid/solid or solid/fluid interface is determined by a complex interplay of the effects of initial or pre-stress and gravity and is distinguished from the classical Rayleigh-Taylor instability in stratified fluids by the existence of a finite critical wavelength. Equivalently, there exists a critical stress at which an interfacial perturbation of a given wavelength will become unstable.

For an unstressed elastic half-space with a shear modulus $G$ and a density exceeding that of a fluid substratum by some positive $\Delta \rho$, the critical (unstable) wavelength is given by $4 \pi G / \Delta \rho g$. For typical values of $G \approx 10^{9} / 10^{11} \mathrm{MPa}, \Delta \rho \approx 500 \mathrm{~kg} \mathrm{~m}^{-3}, g=$ $10 \mathrm{~m} \mathrm{~s}^{-2}$ one finds $L \approx 10^{6} / 10^{8} \mathrm{~m}$. The fact that these values are far in excess of any relevant wavelength even for geological systems illustrates the importance of the destabilizing effects of pre-stress and material softening, both of which are found to be capable of reducing critical wavelengths drastically. In addition, there is the effect of a finite layer thickness, which will have to be taken into account whenever critical wavelengths reach comparable magnitudes.

The instability of the interface between an elastoplastic layer and a buoyant fluid substratum will be promoted both by lateral compression and extension, if the layer stiffness is thereby reduced.

The post-bifurcation behaviour of a destabilized, evolving interface in a two-layer solid/fluid or solid/solid system remains to be studied. A particularly important question concerns the nature of stable finite-amplitude disturbances and the conditions under which they will exist in layers of finite stiffness.

\section{ACKNOWLEDGEMENTS}

This paper was published with the permission of Shell Research B.V. We thank Dr Y. Leroy for helpful discussions and suggestions. 


\section{REFERENCES}

Biot, M. A.

Biot, M. A.

BIOT, M. A.

BIOT, M. A.

BUDIANSKY, B.

ChandRasekHaR, $\mathrm{S}$.

ChandRaseKHAR, $\mathrm{S}$.

DANEŠ, Z. F.

HILL, R.

Hit., R. and Hutchinson, J. W.

HUTCHINSON, J. W.

KOITER, W. T.

RAMBERG, H. R.

1981

RICE, J. R.

SELIG, F.

STÖREN, S. and Rice, J. R.

TAYLOR, G. I.

YOUNG, N. J. B. 1963a Proc. R. Soc. London A273, 306.

1963 b Proc. R. Soc. London A273, 329.

1963 c Proc. R. Soc. London A273, 340.

1965 Mechanics of Incremental Deformation. Wiley, New York.

1974 In Advances in Applied Mechanics (edited by C. S. Y IH), Vol. 14, pp. 1-66. Academic Press, New York.

1955 Proc. Cambr. Phil. Soc. 51, 162.

1961 Hydrodynamic and Hydromagnetic Stability. Oxford University Press, Oxford.

1964 Geophysics 29, 414.

$1958 \quad J$. Mech. Phys. Solids 6, 236.

1975 J. Mech. Phys. Solids 23, 239.

1974 In Advances in Applied Mechanics (edited by C. S. YıH), Vol. 14, pp. 1-66. Academic Press, New York.

1945 On the Stability of Elastic Equilibrium. Doctoral Dissertation, Delft Technology University, Delft, The Netherlands. (English Translation: NASA Report TTF-10, 1967.)

Gravity, Deformation and the Earth's Crust (second edition). Academic Press, London.

1976 Theoretical and Applied Mechanics. Proc. 14th IUTAM Conference (edited by $W$. T. KoITER), pp. 207-220. North Holland, Amsterdam.

1965 Geophysics 30, 633.

1975 J. Mech. Phys. Solids 23, 421.

1950 Proc. R. Soc. London A201, 192.

1976 J. Mech. Phys. Solids 24, 77. 